\title{
Periodismo colmena en la Facultad de Comunicación de la Universidad de Sevilla: Proyecto de investigación periodística colaborativo y multimedia sobre acoso escolar
}

LORENA R. ROMERO-DOMÍNGUEZ

Universidad de Sevilla

Facultad de Comunicación

Departamento de Periodismo II

lorenaromero@us.es

ORCID: https://orcid.org/0000-0001-6942-0183

D.O.I.: http://dx.doi.org/10.12795/JDU.2018.i01.38

Pp.: 671-694

\section{Resumen}

La presente comunicación aborda los resultados del ciclo de mejora implementado en la asignatura Técnicas de Investigación en el Periodismo. Se explican en ella los principios didácticos sintetizados tras la experiencia en la Red de Formación e Innovación Docente (REFID), con especial atención a los siguientes elementos: mapa de contenidos y problemas clave, modelo metodológico con secuencia de actividades y herramientas de evaluación. 
Palabras clave: Técnicas de Investigación en el Periodismo, Grado en Periodismo, Periodismo Colmena, Docencia Universitaria, Experimentación Docente Universitaria.

\section{Breve descripción del contexto de la intervención}

La asignatura de Técnicas de Investigación en el Periodismo se imparte en el segundo cuatrimestre del tercer curso del Grado en Periodismo y del Doble Grado en Periodismo y Comunicación Audiovisual. Es una asignatura de carácter obligatorio en la cual hay cuatro grupos (dos de mañana y dos de tarde) impartidos, en total, en el presente curso académico 2017-2018 por seis profesores. Entre los cuatro grupos del Grado en Periodismo hay 319 alumnos matriculados y, en el Doble Grado en Periodismo y Comunicación Audiovisual, 18, lo que hace un total de 337 estudiantes. Solo se ha implantado el ciclo de mejora que describimos en la presente comunicación en el Grupo 1, donde hay 61 alumnos matriculados del Grado en Periodismo y 13 del Doble Grado en Periodismo y Comunicación Audiovisual, teniendo un total de 74 alumnos en el presente curso académico..

\section{Diseño del CM}

\section{Mapa de contenidos y problemas claves}

En Técnicas de la Investigación en el periodismo pretendemos, además de formar al alumno en contenidos, estimularlo personal y profesionalmente, desarrollando su capacidad de análisis e incentivando su espíritu crítico ante los procesos periodísticos. La consecución de esta doble meta orienta nuestra actividad docente y así lo hemos tenido en cuenta para la determinación de nuestros 
objetivos didácticos y la elaboración del mapa de contenidos con los principales problemas claves que estructuran el sentido de las clases, además del tiempo que transcurre entre ellas.

Coincidimos con González García en defender la aportación al aprendizaje significativo que permite el mapa conceptual, definido como "una representación visual de la jerarquía y las relaciones entre conceptos contenidas por un individuo en su mente" (2008, p.52). Esta estructuración coherente y ordenada adquiere todo su valor cuando los distintos elementos interactúan entre sí y construyen una red de significado. También debemos tener en cuenta que es solo una de las posibles representaciones de una estructura conceptual, siendo un elemento flexible y abierto a la reelaboración a lo largo del cuatrimestre.

Los bloques temáticos contenidos en el Proyecto Docente de la asignatura se han sintetizado en el siguiente mapa conceptual.

Jornadas de Formación e Innovación Docente del Profesorado I № 1 (2018) Esta obra se distribuye con la licencia Creative Commons 


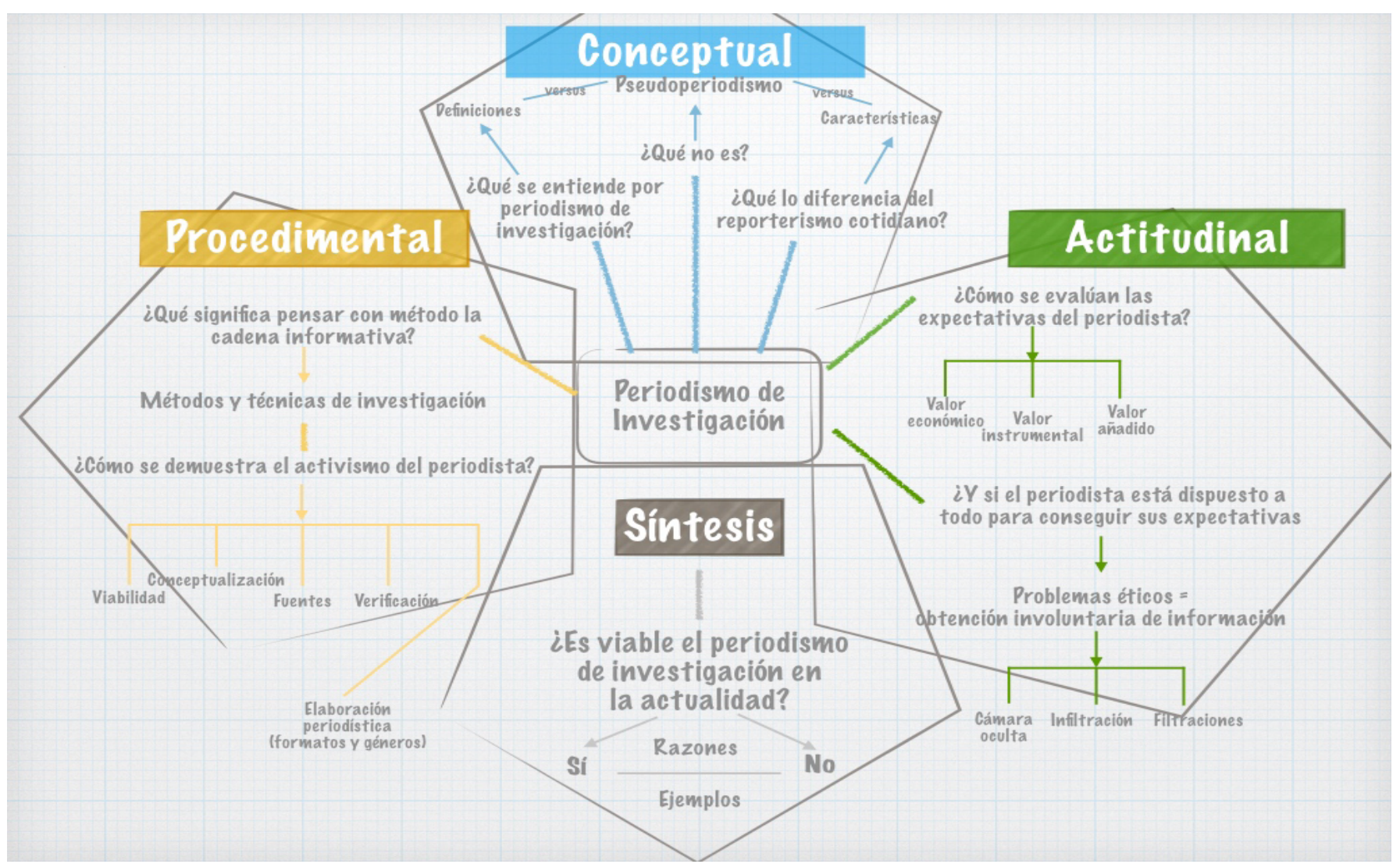

Figura 1. Mapa conceptual de Técnicas de Investigación en el Periodismo

Jornadas de Formación e Innovación Docente del Profesorado | № 1 (2018)

(C) Esta obra se distribuye con la licencia Creative Commons

cc) (1) $\Theta$ Reconocimiento-NoComercial-SinObraDerivada

Internacional (CC BY-NC-ND 4.0.) 


\section{Modelo metodológico posible}

Los modelos repetitivos no resultan útiles en nuestra materia desde el momento en que: 1) Pretendemos enseñar conceptos complejos (ética, honestidad profesional, violencia contra la libertad de información); 2) Pretendemos enseñar procedimientos complejos (protocolos de búsqueda de fuentes documentales y orales que escapan a las voces oficiales del periodismo de declaraciones imperante); 3) Pretendemos desarrollar actitudes y valores que en muchas ocasiones no son predominantes en el entorno mediático actual (diligencia, compromiso social, etc.). Y utilizamos el término 'complejo' en el sentido que lo define Morin en su obra Siete saberes necesarios para la educación del futuro: "un tejido interdependiente, interactivo e interretroactivo entre el objeto de conocimiento y su contexto." (1999, p.17)

Todas estas cualidades definen un modelo de enseñanza/aprendizaje de carácter no lineal donde se requiere activismo mental del alumno, confrontación de informaciones con sus ideas previas y resolución de la situación problemática en unas conclusiones finales compartidas por el grupo (De Alba y Porlán, 40-41). El entorno de aprendizaje en el cual impartimos la asignatura se sustenta sobre un modelo de carácter investigativo (que definimos como idóneo durante el pasado Curso de Iniciación), donde se suceden las siguientes fases:

1. SP. Situación Problemática: Planteamos un problema que dé sentido al proceso de aprendizaje para motivar la reflexión de los estudiantes y estimular la adquisición de conocimiento autónomo, a través de estrategias diversas en el aula, como: definición de problemas de investigación desde la metodología colaborativa y resolución de los mismos, talleres conceptuales, exposiciones, tutorías colectivas para aplicar estrategias de co- y auto-evaluación, control de lecturas 
obligatorias, disponibles en la Plataforma Virtual, seminarios impartidos por profesionales del periodismo. De este modo, se potencia la intervención de periodistas en el aula.

2. E1. Facilitar la expresión de los modelos iniciales de los estudiantes, sacando a la luz sus esquemas mentales a través de la exposición pública y debates.

3. T-P. Contenido Teórico Práctico: Presentación de conceptos (teóricos, procedimentales y actitudinales) apoyándonos, en todo momento, en el uso de soportes hemerográficos y audiovisuales disponibles para los alumnos en las guías didácticas publicadas en la Plataforma Virtual al comienzo de cada unidad.

4. E2. Formulación del nuevo modelo de los estudiantes. El estudiante pueda re-elaborar sus conocimientos y exponer las ideas adquiridas tras su participación en las actividades propuestas, aplicándolas de manera directa a su proyecto de investigación

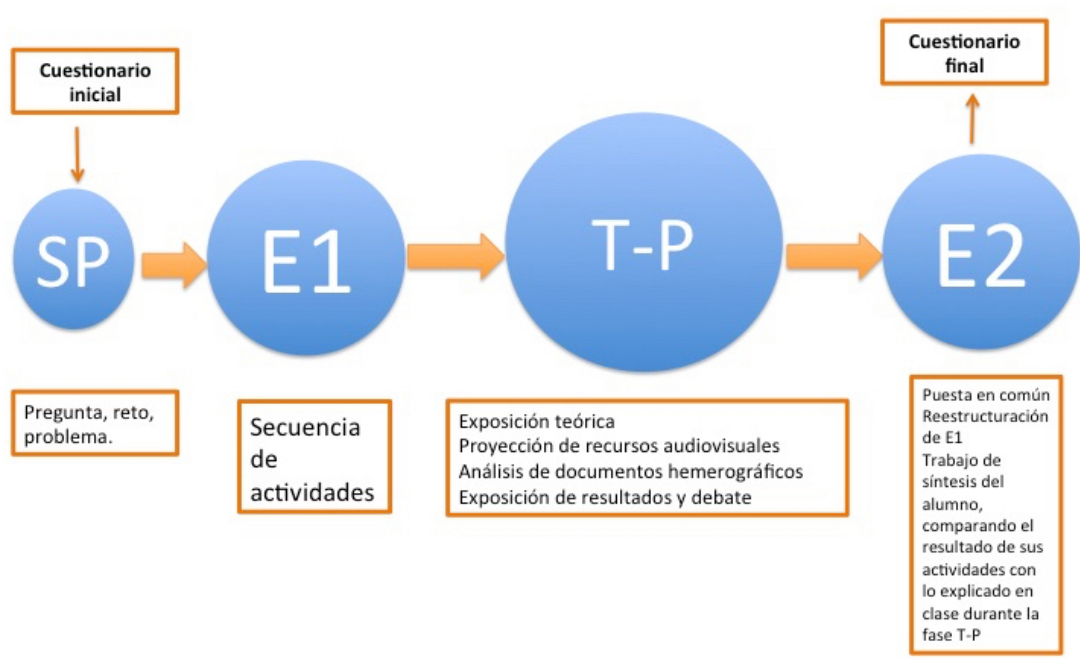

Figura 2. Modelo metodológico

Jornadas de Formación e Innovación Docente del Profesorado | № 1 (2018) Reconocimiento-NoComercial-SinObraDerivada $\quad 4.0$ Internacional (CC BY-NC-ND 4.0.) 


\section{Secuencia de actividades}

\section{SEMANA 1}

TEMA2. EL "MÉTODO” EN LA INVESTIGACIÓN PERIODÍSTICA. 2.1. Cómo empezar una investigación.

\section{Actividad 1}

Título: "Hoja de ruta" à Tipo de realización: Grupal à Tiempo de realización: 30 minutos diseño inicial + 60 minutos explicación docente con ejemplos de casos reales + 30 minutos de reflexión y corrección del diseño inicial, redactando uno nuevo à Instrucciones: El alumno debe diseñar una línea de tiempo donde se visualicen las distintas tareas que cree va a desarrollar hasta el final del cuatrimestre para poder abordar su investigación periodística. Una vez finalizado, cada subgrupo deberá exponer sus etapas, procediéndose a apuntar en la pizarra cada uno de los momentos señalados por los estudiantes durante sus intervenciones. El resultado obtenido se contrastará con el esquema desarrollado por Pepe Rodríguez en su obra Periodismo de investigación: técnicas y estrategias

Cada subgrupo deberá volver a su cronograma anotando las etapas que no había contemplado e indicando de manera razonada por qué no lo había tenido en cuenta y si considera útil las indicaciones contenidas en el cuadro del autor.

2.2. Lo público y lo privado: la "ley del silencio".

\section{Actividad 2}

Título: "Tabú primero de España" à Tipo de realización: individual / fuera del aula à Tiempo de realización: El que cada alumno necesite para la lectura del material propuesto, así como para la búsqueda de ejemplos que confirmen sus argumentos sobre la existencia de temáticas vetadas en los medios de comunicación como consecuencias de los intereses ajenos a los criterios periodísticos. La presentación de resultados sí se hará dentro del horario de clase en un máximo de 30 minutos. Esta exposición pública permitirá contrastar los resultados obtenidos 
y definir similitudes con otros casos propuestos por la docente à Instrucciones: Tomando como referencia algunas de las portadas publicadas por la publicación satírica El Jueves, el alumno deberá reflexionar sobre cómo ha cambiado el tratamiento mediático de la monarquía en los medios españoles, respondiendo a las siguientes preguntas:

- ¿Por qué nadie se atreve/atrevía a criticar al monarca?

- ¿Por qué no se publica/publicaba información relevante sobre su vida privada o sus negocios?

- ¿Cuál es la dimensión de su fortuna privada?

- ¿Por qué no se publican fotos del rey en postura indecorosa?

- ¿Cuáles son las razones para el pacto de silencio establecido en torno a la monarquía en nuestro país?

Deberá leer, además, el capítulo 11 del libro Cazadores de noticias. Los 'años de oro' del periodismo de investigación en España, de Miguel Ángel Nieto donde se suministran algunos ejemplos recogidos por el autor desde la década de los años 80.

\section{SEMANA 2}

\subsection{El estudio de viabilidad.}

\section{Actividad 3}

Título: "Probando, probando". A través de esta actividad se delimita la viabilidad del proyecto y ayuda a definir el éxito o el fracaso a priori de nuestra investigación à Tipo de realización: Grupal / fuera del aula à Tiempo de realización: 35 minutos visionado + 120 minutos estudio de viabilidad + 60 minutos exposición pública de resultados + 60 minutos corrección plantilla suministrada por docente (estas dos últimas etapas sí se realizan durante el horario de clase) à Instrucciones: Visionado del documental "No abusarás" (disponible en https://www.youtube.com/watch?v=gHICOTN 100) y realización del estudio de viabilidad según esquema contenido en la Guía Didáctica del Tema 2 (disponible en la Plataforma Virtual de la USE). Una vez finalizada, la siguiente sesión se dedicará a que cada subgrupo 
trabaje de manera autónoma su estudio de viabilidad, esta vez aplicándolo a la línea de investigación propuesta dentro del tema genérico del acoso escolar.

\section{SEMANA 3}

Durante toda la semana los alumnos siguen trabajando en clase sus estudios de viabilidad y se introduce la explicación teórica de la docente sobre la definición conceptual de sus proyectos. El trabajo autónomo de cada subgrupo cuenta con la asesoría de la docente.

\section{SEMANA 4}

\subsection{Las fuentes documentales.}

\section{Actividad 4}

Taller impartido por personal de la Biblioteca de la Facultad de Comunicación sobre búsquedas bibliográficas, tipología de recursos documentales, modelos de citas, etc.

Tiempo de realización: 120 minutos. Los compañeros de la Biblioteca finalizan la sesión poniendo algunos ejercicios prácticos para comprobar que el estudiante ha asimilado las explicaciones. [No realizado]

2.5. El trabajo con las fuentes orales.

\section{Actividad 5}

Titulo: "La realidad siempre supera la ficción" à Tipo de realización: Individual / en clase à Tiempo de realización: (Sesión 1) 20 minutos explicación tipología de fuentes + 50 visionado largometraje + 50 minutos identificación de problemas y puesta en común; (Sesión 2) 120 minutos: se intercalan explicaciones teóricas, casos prácticos y reflexión colectiva de alumnos à Instrucciones: Visionado en clase de una secuencia de La sombra del poder (minuto 50 hasta el final del largometraje. Información sobre la película disponible en https://www.filmaffinity.com/es/film600416. html). Se solicita a los alumnos que identifiquen los tipos de fuentes representadas según tipología explicada en clase y que señalen las principales dificultades que sufren los periodistas protagonistas del film en su ejercicio profesional. Las situaciones inidentificadas serán comparadas posteriormente en clase con casos reales expuestos por 
la docente y en los cuales se han detectado problemas similares. Se solicita a los alumnos que intenten esclarecer las consecuencias que esos problemas ha tenido sobre el trabajo periodístico. En esta dinámica se intercalan explicaciones teóricas, presentación de casos prácticos y aportaciones de los alumnos.

\section{SEMANA 5}

TEMA 3. PROBLEMAS ÉTICOS EN EL PERIODISMO DE INVESTIGACIÓN.

3.1. Valor económico, valor instrumental y valor añadido. Actividad 6

Título: "El fin ¿justifica? Los medios" à Tipo de realización: Grupal en clase à Tiempo de realización: 120 minutos à Instrucciones: Se suministran tres ejemplos periodísticos (testimonio de Emilio Suárez Trashorras como confidente para El Mundo en su investigación sobre el 11M; la situación del periodista británico John Cantlie en Siria como informador "al servicio" del Estado Islámico y la película El precio de la verdad, inspirada en la vida del periodista Stephen Glass, para que intenten conectar cada uno de estos ejemplos con los tres valores sobre los cuales se sustenta la evaluación de expectativas de un profesional de la información a la hora de hacer su trabajo. El ejercicio finaliza con la resolución en clase a través de la explicación teórica de cada uno de estos conceptos y la corrección de los alumnos.

3.2. Obtención 'involuntaria' de información.

Se intercalan explicaciones teóricas, casos prácticos y reflexión colectiva de alumnos.

\section{SEMANA 6}

\subsection{La cámara oculta.}

\section{Actividad 7}

Título: "A vueltas con la legalidad" à Tipo de realización: Individual / fuera del aula à Tiempo de realización: 120 minutos aprox. de lectura del material recomendado à Instrucciones: Exposición en clase de resultados de lectura y reflexión sobre las variaciones experimentadas en la 
tipificación legal del uso de la cámara oculta en los reportajes periodísticos. Posterior debate con los alumnos divididos en dos grupos -a favor y en contra del uso de esta herramienta- donde deben aportarse argumentos legales, periodísticos y deontológicos para defender cada una de las posiciones. Se valorará muy positivamente el hecho de que los grupos de trabajo aporten ejemplos de reportajes donde se usa esta técnica.

3.4. Infiltración y suplantación de personalidad.

Estudios de caso: Nelly Bly (10 días en una casa de locos), Günther Wallraff (Negro sobre blanco) y Antonio Salas (El palestino).

Exposición voluntaria por parte de tres alumnos + explicación teórica de la docente.

[No realizado]

\section{SEMANA 7}

\subsection{Las filtraciones periodísticas.}

\section{Actividad 8}

Título: Taller conceptual sobre las filtraciones: De 'Garganta Profunda a John Doe' à Tipo de realización: grupal à Tiempo de realización: 180 minutosà Instrucciones: la clase tiene que dividirse en grupos de 6 personas, máximo y seleccionar algunos de los siguientes casos: Watergate, Papeles del Pentágono, Caso GAL, Wikileaks, Snowden, Falciani, Los papeles de Bárcenas, Panama Papers. El propósito del taller es entender el valor de las filtraciones en el proceso productivo periodístico (nivel 1 de comprensión) y las consecuencias que los casos denunciados en ellas han tenido a nivel legal y político (nivel 2 de comprensión). En este proceso, también se aprenderán a distinguir las filtraciones "reales" de las "deliberadas", además de comprender la evolución sufrida por los distintos actores involucrados en un proceso de filtración desde los tiempos del Watergate a la actualidad de investigaciones como los Papeles de Panamá.

Parte I. Cada grupo deberá abordar el caso seleccionado respondiendo a las siguientes preguntas: 
- ¿Han puesto en jaque a los servicios de inteligencia de Estados democráticos?

- ¿Cuestionan la legalidad vigente en materia de secretos por su carácter general?

- ¿Son una respuesta de voces no autorizadas (whistleblowers) ante supuestas mentiras oficiales?

- ¿Utilizan métodos informáticos sofisticados para realizar la filtración?

- ¿Han contado con la simpatía popular por revelar informaciones que alertan sobre abusos de poder y corrupción?

- ¿Han tenido eco en medios de difusión masiva o en medios de carácter alternativo?

- ¿Ha puesto en funcionamiento la maquinaria de verificación de los medios que se han hecho eco de ellas?

- ¿Han provocado crisis políticas y dimisiones?

- ¿Ha habido consecuencias legales para los filtradores?

Parte II. El grupo debe sintetizar los resultados del análisis de la Parte I en la siguiente tabla:

\begin{tabular}{|c|c|c|c|c|c|c|c|}
\hline Caso & $\begin{array}{l}\text { Canal de } \\
\text { filtración }\end{array}$ & $\begin{array}{l}\text { Fuente } \\
\text { anónima/ } \\
\text { conocida }\end{array}$ & Medio & $\begin{array}{l}\text { Noticias } \\
\text { generadas }\end{array}$ & $\begin{array}{l}\text { Consecuencia } \\
\text { para periodistas }\end{array}$ & $\begin{array}{l}\text { Consecuencia } \\
\text { para filtradores }\end{array}$ & $\begin{array}{l}\text { Consecuencias } \\
\text { legales y políticas }\end{array}$ \\
\hline \multicolumn{8}{|c|}{ Watergate } \\
\hline \multicolumn{8}{|c|}{ Pentágono } \\
\hline \multicolumn{8}{|l|}{ GAL } \\
\hline \multicolumn{8}{|l|}{ Wikileaks } \\
\hline \multicolumn{8}{|l|}{ Snowden } \\
\hline \multicolumn{8}{|l|}{ Falciani } \\
\hline \multicolumn{8}{|l|}{ Bárcenas } \\
\hline Panamá & & & & & & & \\
\hline
\end{tabular}

En el ejercicio de sintesis deberéis discutir los resultados y organizarlos en torno a las siguientes cuestiones:

- ¿Es la filtración un recurso habitual de las fuentes? 
- ¿En qué medida se hacen eco los periodistas de la información filtrada?

- ¿Cómo se garantiza la fiabilidad de una fuente que filtra?

- ¿Es posible distinguir entre el filtrador real y el filtrador simulado?

- ¿Cómo controla el periódico la veracidad de los datos filtrados?

- ¿Quiénes son los beneficiarios y benefactores de este tipo de recursos?

- ¿Qué intereses ocultos respaldan esta política por parte de los medios?

- ¿Cómo influye en la configuración de la actualidad una información producto de filtraciones?

- ¿Es la audiencia consciente del grado de desinformación a la que se encuentra expuesta?

Parte III. Puesta en común de los resultados de todos los casos para completar de manera colectiva la tabla de análisis.

3.6. El secreto profesional.

Estudio de caso: Análisis del concepto y el uso del secreto profesional a través del caos conocido como Plamegate.

Tiempo de realización: 60 minutos. [No realizado]

\section{SEMANA 8}

TEMA 4. PERIODISMO DE INVESTIGACIÓN Y PODER.

4.1. Los enemigos del periodismo de investigación.

Explicación teórica tomando como punto de partida casos reales sobre los obstáculos con los cuales se enfrenta la investigación periodística. La segunda parte de la exposición se centra en cómo estos obstáculos se transforman en violencia directa contra los periodistas. Entendemos el concepto de "violencia" desde una perspectiva muy amplia, y así se le transmite al alumno, haciéndole ver que no sólo países con regímenes dictatoriales o en vías de desarrollo son los únicos enclaves donde se silencia la labor de los periodistas, sino que también en el mundo 
occidental y desarrollado son muchas las estrategias que consiguen similares resultados: silenciar a los periodistas.

Tiempo: 120 minutos

4.2. Las rutinas de producción periodística y la precariedad laboral

\section{Actividad 9}

Taller impartido por Sindicato de Periodistas de Andalucía / Colegio Profesional de Periodistas de Andalucía para reflexionar sobre la situación del sector y posterior debate con los alumnos.

Tiempo: 120 minutos.

[No realizado]

\section{SEMANA 9}

4.3. Los hombres de negocio en los medios; 4.4. La publicidad; 4.5. La connivencia con la política y el poder del Estado

\section{Actividad 10}

Taller impartido por periodistas de Fundación Civio

Tiempo: 120 minutos.

[No realizado]

\section{SEMANA 10}

4.6. ¿Es viable el Periodismo de Investigación?

Exposición de resultados de los trabajos de los subgrupos, con especial atención a la valoración de las fuentes entrevistadas, los objetivos alcanzados y las dificultades encontradas en su ejercicio como profesionales de la información. Este trabajo de sintesis final debe ayudar a estimular un debate sobre la necesidad o no del Periodismo de Investigación en nuestra sociedad actual, reflexionando sobre si es una alternativa a la crisis de la profesión.

\section{Evaluación}

Técnicas de Investigación en el Periodismo ofrece un sistema donde el alumno puede decidir si quiere formar parte de un proceso de evaluación colectiva y continua (Opción A) o si prefiere orientarse solo a obtener una calificación individual (examen y prácticas). Hemos incluido 
en la opción colectiva también un ejercicio de carácter individual porque, en consonancia con García Irles, Sempere Ortells, Marco de la Calle y De la Sen Fernández (2011b, 1816) entendemos que "la responsabilidad individual y la interdependencia grupal, son condiciones del aprendizaje colaborativo" y no cualidades excluyentes.

\begin{tabular}{|c|c|c|}
\hline \multirow[b]{2}{*}{ OPCIÓN A } & $\begin{array}{l}\text { Examen } 40 \% \\
\text { (* Porcentaje } \\
\text { revisable) }\end{array}$ & $\begin{array}{l}\text { - Preguntas lecturas obligatorias y material ana- } \\
\text { lizado en aula o de manera autónoma por } \\
\text { alumno } \\
\text { - } \text { Batería de preguntas cortas }\end{array}$ \\
\hline & $\begin{array}{c}\text { Proyecto de } \\
\text { Investigación } \\
\text { Colaborativo } \\
\text { Multimedia } \\
60 \%\end{array}$ & $\begin{array}{l}\text { - Temática común a toda la clase, con subáreas } \\
\text { de trabajo } \\
\text { - Trabajo grupal. } \\
\text { - Durante el curso y hasta el } 31 \text { de mayo cada } \\
\text { grupo debe haber publicado en el blog: } \\
\text { - Fotorrelato (0,5 puntos) } \\
\text { - Cortes audiovisuales de entrevistas reali- } \\
\text { zadas a fuentes ( } 1 \text { punto) } \\
\text { - Desarrollo estadístico e infográfico de da- } \\
\text { tos (0,5 punto) } \\
\text { - Entrevistas escritas (1 punto) } \\
\text { Entregas: } \\
\text { - Estudio de viabilidad (1 punto). } 22 \text { de } \\
\text { marzo de } 2018 . \\
\text { - Fuentes (1 punto): } 31 \text { de mayo de } 2018 \text {. } \\
\text { Memoria ( } 1 \text { punto): } 25 \text { de junio de } 2018 \text { du- } \\
\text { rante el horario del examen. }\end{array}$ \\
\hline
\end{tabular}

Explicación del Proyecto de Investigación Colaborativo Multimedia tomando como referencia los siguientes casos reales: The new arrivals; Medicamentalia y Obama's secret struggle to punish Russia for Putin's election assault. La idea de esta modalidad es que el alumno sea capaz de adquirir los contenidos procedimentales y actitudinales básicos para poder elaborar por sí mismo un reportaje de investigación. Partimos de la premisa de que no se puede investigar si no se compromete al alumno con un tema que le sea interesante y relevante. Solo a través de la práctica diaria se puede adquirir una rutina de trabajo que le acerque a esta disciplina periodística. Por ello, las clases serán consideradas como grandes redacciones 
interconectadas donde los alumnos van a evidenciar dinámicas de trabajo propias de los profesionales en la actualidad (trasladaremos al aula -en sentido real y simbólico, dado que los alumnos deben desarrollar buena parte de su investigación fuera de este espacio- los conceptos propios de lo que se conoce hoy en día como el "periodismo colmena").

Han sido 55 los alumnos que se han sumado a la Opción A. De los 19 alumnos restantes matriculados en el Grupo 1 del Grado en Periodismo y del Doble Grado en Periodismo y CAV solo 6 han marcado la opción B. El resto de estudiantes (13) se encuentran en programas de movilidad durante el cuatrimestre o no han venido a clase por problemas de incompatibilidad horaria, excluyéndose automáticamente su participación en el Proyecto.

El tema se ha decidido de manera colectiva en las primeras semanas de clase: los alumnos han entregado sus fichas rellenas y ha salido finalmente el tema del acoso escolar. El proyecto se realiza de manera grupal y se han creado, a su vez, equipos de trabajo que abordan cada una de las líneas que han planteado en una primer documento de toma de contacto, donde han manifestado sus inquietudes como ciudadanos y como futuros periodistas. Los equipos de trabajo se han conformado atendiendo a los propios intereses de los alumnos y cuentan con un número variable de estudiantes en función de la complejidad del subtema. Todo el material está alojado en el siguiente blog: https://tecnicasdeinvestigacionenelperiodismo.wordpress.com

Hemos establecido varios momentos en la evaluación:

e) Fase previa: donde se pueden conocer y evaluar las expectativas de los estudiantes sobre la materia, con actividades conducentes a comprobar qué entienden por Periodismo de Investigación, cuáles son sus áreas de interés, etc. Durante la primera semana planteamos una actividad introductoria sobre el denominado "Periodismo

Jornadas de Formación e Innovación Docente del Profesorado I № 1 (2018) Esta obra se distribuye con la licencia Creative Commons 
colmena" a través del estudio de casos, que deben poner en relación con las lecturas The back door, Crowdsourcing in investigative journalism, Nuevas narrativas informativas: El caso de Storify y Renovarse o morir. Los 'docugames', una nueva estrategia transmedia que reinventa las formas de transmitir la realidad. Gracias a este ejercicio, podemos conocer y evaluar las nociones previas y expectativas de los estudiantes, siendo estos datos de vital importancia para la selección de los ejercicios que se desarrollarán durante el cuatrimestre.

f) Fase intermedia: enfocada al proceso del aprendizaje, no tanto a la obtención del producto final (el reportaje de investigación albergado en el blog), tiene un carácter formativo permitiendo la reelaboración de las entregas que se realizan a lo largo del cuatrimestre con el feedback y el feedforward de las correcciones colectivas realizadas en clase y de manera individualizada a través de la acción tutorial. De este modo, el alumno no solo obtiene información sobre los fallos sino, lo más importante, "sobre los caminos para superarlos" (Georgeta, Silva y Cano García, 2013). En este sentido, la herramienta más interesante para observar la evolución de los alumnos es la memoria de trabajo o diario del alumno, que se entrega el día de la prueba final de evaluación y que contiene una explicación del proceso de investigación realizado, señalando obstáculos encontrados, grado de demostración de objetivos/hipótesis de trabajo, aportaciones realizadas a la temática propuesta y breve autoevaluación. Dada la fecha de entrega de la presente comunicación no podemos aportar la valoración de dicho documento, pues la entrega del mismo se producirá el 25 de junio, 
día en el cual está fijada la prueba de evaluación de la asignatura. No obstante, sí podemos aportar nuestra reflexión en torno al ejercicio de feedforward que hemos realizado con la entrega 1, la referida al estudio de viabilidad y la definición conceptual del proyecto de investigación. Tras la corrección inicial de dicho documento que, recordemos, había sido elaborado en buena parte durante el horario de clase, los distintos equipos de trabajo recibieron en su correo un documento orientativo con las mejoras a introducir para poder acometer el proyecto de investigación según las directrices de la docente. De los 20 equipos de trabajo, 13 han vuelto a remitir el documento con las mejoras incorporadas lo que ha permitido avanzar en el proceso de investigación de manera más satisfactoria para todos ellos.

g) Fase final: De carácter sumativo, se realiza al final del curso y valora los resultados obtenidos (tanto en el proyecto como en la prueba final, además de atender a otros criterios como la asistencia, la participación, la progresión) en relación con las competencias y los aprendizajes obtenidos. En este sentido planteamos en la prueba final de reflexión preguntas similares a las planteadas al comienzo del cuatrimestre para conocer la evolución de los estudiantes y si han modificado sus ideas previas sobre la asignatura.

\section{Aplicación del Ciclo de Mejora Docente}

Hemos optado por presentar un relato sintético de las sesiones de clase durante las cuales se ha implementado el Ciclo de Mejora, teniendo en cuenta la duración del mismo (40 horas), la similitud de las situaciones experimentadas y la limitación de espacio del presente trabajo. 
La primera aclaración que deseamos realizar es que el grupo ya ha cursado una asignatura con la profesora en el primer cuatrimestre (Periodismo Político y Económico, para la cual diseñamos nuestros dos primeros ciclos de mejora durante la fase de iniciación), lo que ha permitido un grado de conocimiento y familiaridad que nos ha capacitado para abordar de manera muy directa los numerosos problemas que hemos experimentado durante el desarrollo del segundo cuatrimestre.

Estas situaciones conflictivas se han planteado, fundamentalmente, en las dificultades experimentadas en los equipos de trabajo para llevar el trabajo organizado de manera semanal, cumpliendo con las etapas definidas en la elaboración del proyecto de investigación. De hecho, podemos afirmar, por el aumento en el flujo de intercambio de correos, así como por las visitas a tutorías individuales fuera del tiempo de clase, que la mayoría de los equipos han concentrado buena parte de sus trabajos en las cuatro semanas previas al cierra del blog. Esta ha sido una de las principales dificultades experimentadas, dado que la ausencia de compromiso por parte de los alumnos desde la primera sesión ha marcado un ritmo frenético durante las últimas clases que no han sido propicias para generar un clima de trabajo agradable. De este modo, se han sucedido varias sesiones donde se ha tenido que volver a explicar contenidos ya vistos en el inicio del cuatrimestre y otras en las cuales se ha tenido que incidir en el carácter progresivo del trabajo y la evolución como elementos importantes en la evaluación del alumno, quien se ha mostrado muy confundido pensando que solo se le iba a examinar por los contenidos subidos al blog. De este modo, podemos afirmar que, realmente, el planteamiento de la experiencia de evaluación a través del proyecto colaborativo multimedia ha ocasionado más extrañeza que entusiasmo, así como algún que otro momento de pánico colectivo ante la ausencia de material propio obtenido tras la adquisición de las pautas durante las sesiones de clase.

Podemos afirmar que ha habido una excesiva focalización en las piezas periodísticas subidas al blog, desatendiendo los elementos más formativos como su capacidad 
para resolver situaciones problemáticas y buscar alternativas y debemos dudar realmente de haber conseguido un entorno de trabajo colaborativo ya que los alumnos han repetido material y fuentes de información cuando podrían haberlo compartido sin necesidad de duplicarlo.

Las clases dedicadas a tutorías colectivas para la resolución de dudas o la elaboración de determinadas partes del proyecto (estudio de viabilidad y definición conceptual, fundamentalmente) no han involucrado a todos los equipos de trabajo y ha habido un ritmo muy descompensado entre equipos que han utilizado todo el tiempo de clase para plantear sus inquietudes sobre el trabajo y otros que no han traído, si quiera, un esquema sobre el que poder plantear interrogantes.

En cuanto a las actividades planteadas nos hemos encontrado una situación muy similar a la descrita en nuestros anteriores ciclos del mejora de la etapa de iniciación: el interés desmedido solo por aquellas actividades gracias a las cuales pueden obtener puntuación para la nota final de la asignatura, la pérdida de tiempo para que formen los grupos de trabajo; la repetición de las misma personas participantes en las actividades de introducción en cada uno de los epígrafes; etc.

\section{Evaluación del Ciclo de Mejora Docente}

Señalamos a continuación las cuestiones que se mantienen y aquellas sobre las cuales debemos seguir trabajando para introducirlas en futuros ciclos:

- Partir de una situación problemática porque es una manera activa de organizar la clase y permite conectar con su propia experiencia y con su futura situación profesional.

- Mantener el mapa de contenidos porque resulta útil para clarificar contenidos, tipos, y relaciones entre ellos. Como mejora de este aspecto, planteamos la posibilidad de crear mapas conceptuales con alumnos para próximos cursos, 
- Idoneidad de la evaluación con feedforward, extensible a todas las entregas realizadas por los alumnos durante el cuatrimestre.

- Apostar de manera más decidida por las sesiones de co-evaluación en el aula para que el alumno pueda visualizar de manera inmediata las mejoras que es posible introducir en su trabajo para adquirir las competencias marcadas en el proyecto de investigación.

- Diversificar las herramientas de evaluación y secuenciarlas a lo largo del cuatrimestre (al principio, durante y al final de la asignatura).

- Intentar definir rúbricas de evaluación en colaboración con los alumnos, sin perjuicio de herramientas más tradicionales orientadas hacia los alumnos que no desean implicarse en el proyecto de investigación colaborativo multimedia.

- Estimular a los alumnos para que todos participen en la experiencia del proyecto colaborativo multimedia, eliminando, de este modo, el examen al final del cuatrimestre.

De este modo, y en consonancia con los principios didácticos que ya definimos durante el curso de iniciación, seguimos manteniendo la idoneidad de los mismos:

\section{Qué debe saber el estudiante}

a) Los mapas conceptuales son herramientas fundamentales que dotan de sentido el temario de una asignatura, percibiéndola de forma global en su relación con otras materias.

b) Las clases deben atender a los contenidos conceptuales, procedimentales y actitudinales de elevada dificultad, dado que el alumno puede por sí solo adquirir datos de bajo nivel de manera autónoma. 
a) El modelo metodológico debe incluir siempre un apartado dedicado a exponer y reflexionar sobre las ideas previas de los alumnos, ya que ello nos ayuda a ajustar el nivel de la clase y a conectar con ella.

b) El planteamiento de problemas clave ayuda a "enganchar" al alumno y darle un motivo para seguir atento durante todo el desarrollo de la sesión.

c) La secuencia de actividades contenida en el modelo metodológico debe ser coherente y clara en su relación con los contenidos que se van a trabajar en el aula.

d) Dinamizar la clase no debe ser el sentido único de las actividades, sino que tienen que responder a las expectativas de los estudiantes y a la del propio docente en lo que respecta a la utilidad de la materia.

\section{Qué, cómo y cuándo evaluar}

a) La evaluación debe contemplar ítems que representen de manera clara y precisa todos los contenidos trabajados en el aula.

b) La evaluación debe estar sistematizada e incluir un número limitado de elementos posicionados en los momentos inicial, intermedio y final del cuatrimestre.

c) El sistema de evaluación diseñado debe permitir ofrecer una retroalimentación rápida al alumno sobre los ejercicios realizados, porque ello permite mejorar el aprendizaje. 


\section{Referencias bibliográficas}

Bain, K. (2007). Lo que hacen los mejores profesores de universidad. Valencia: Universidad de Valencia.

Caccavo, F. (2010). Teaching undergraduates to think like Scientists. College Teaching, 57, 9-14, https://doi. org/10.3200/CTCH.57.1.9-14

De Âlba, N. y Porlán, R. (2017). La metodología de enseñanza. En Porlán, R. (coord.), Enseñanza universitaria. Cómo mejorarla (37-53). Madrid: Morata.

Domingo Segovia, J. (2009). Modelos y estructura del proyecto Docente. En Curso Bases para la elaboración del proyecto docente. Universidad de Granada. Recuperado de http://calidad.ugr.es/pages/ secretariados/form apoyo calidad/docs/materiales-iniciacion/proyecto-docente/presentaciones/ presentacionmodelosestructural

Finkel. D. (2008). Dar clase con la boca cerrada. Universitat de València: Servei de Publicacions.

García Pérez, F. F. (2000). Un modelo didáctico alternativo para transformar la educación: el modelo de investigación en la escuela. Scripta Nova, Revista Electrónica de Geografía y Ciencias Sociales (64), 1-24. Recuperado de http://www.ub.edu/geocrit/sn-64.htm

Georgeta, I., Silva, P., y Cano García, E. (2013). El feedback y el feedforward en la evaluación de las competencias de estudiantes universitarios. Profesorado. Revista de Currículum y Formación de Profesorado, 17(2), 283-301. Recuperado de http://www.redalyc.org/ pdf/567/56729526017.pdf

González, F. (2008). El mapa conceptual y el diagrama V. Recursos para la Enseñanza Superior en el siglo XXI. Madrid: Narcea.

Guisasola, J. y Garmendia, M. (eds.). (2014). Aprendizaje basado en problemas, proyectos y casos diseño e implementación de experiencias en la universidad. Universidad del País Vasco: Secretariado de Publicaciones. Recuperado de https://web-argitalpena.adm.ehu. es/pdf/USWEB149592.pdf

Leal, R. y Zayas, F. (2015). Secuencia didáctica "Quien sabe hablar del camino es porque andado lo tiene". Tejuelo, 22(1), 71-93. Recuperado de http://dehesa.unex.es/ handle/10662/4928

Morales Vallejo, P. (2006). Implicaciones para el profesor de una enseñanza centrada en el alumno. Miscelánea Comillas. Revista de Teología y Ciencias Humanas, 64(124), 
11-38. Recuperado de http://revistas.upcomillas.es/index.php/miscelaneacomillas/article/view/6553

Morin, E. (1999). Siete saberes necesarios para la educación del futuro. UNESCO. Recuperado de http://unesdoc.unesco.org/images/0011/001177/117740so.pdf

Pastor del Pino, M. C. (2011). La evaluación del aprendizaje grupal en la docencia universitaria. En Tortosa Ybáñez, M. T., Álvarez Teruel, J. D. y Pellín Buades, N. (coords.), IX Jornadas de Redes de Investigación en Docencia Universitaria: diseño de buenas prácticas docentes en el contexto actual (1707-1718). Universidad de Alicante. Recuperado de https://rua.ua.es/dspace/ handle/10045/19885

Rodríguez Izquierdo, R. M. (2012). Aplicación de las guías didácticas de trabajo como apoyo al aprendizaje autónomo del estudiante en la asignatura Didáctica en Educación Social. UPO INNOVA: revista de innovación docente (1), 471-492. Recuperado de https://www.upo. es/revistas/index.php/upoinnova/article/view/123

Sempere Ortells, J. M., García Irles, M., Marco de la Calle, F. M. y De la Sen Fernández, M. I. (2011a). Aprendizaje colaborativo: un reto para el profesor en el nuevo contexto educativo. En Gómez Lucas, M. C. y Álvarez Teruel, J. D. (coords.), El trabajo colaborativo como indicador de calidad del espacio europeo de educación superior (89-103). Universidad de Alicante. Recuperado de https://rua.ua.es/dspace/ bitstream/10045/25830/3/art\%C3\%ADculo\%20redes\%202011.pdf

(2011b). La rúbrica de evaluación como herramienta de evaluación formativa y sumativa. En Tortosa Ybáñez, M. T., Álvarez Teruel, J. D. y Pellín Buades, N. (coords.), IX Jornadas de Redes de Investigación en Docencia Universitaria: diseño de buenas prácticas docentes en el contexto actual. Universidad de Alicante. Recuperado de https://rua.ua.es/dspace/handle/10045/19885 